\title{
Paediatric gallstones and laparoscopic cholecystectomy
}

\author{
D. G. Samuel, N. N. Naguib, A. Y. Izzidien
}

Prince Charles Hospital, Merthyr Tydfil, UK; daisams@doctors.org.uk

Received 19 October 2009; revised 30 November 2009; accepted 2 December 2009.

\begin{abstract}
Introduction: Gall stones disease is a rare occurrence in paediatric patients and the diagnosis is often overlooked. Patients often present with non-specific symptoms of abdominal pain and the classic features of gallstones are sometimes absent [1]. The aim of our study is to increase the awareness of cholecystitis and acute pancreatitis being a possible occurrence in the paediatric age group and should therefore be in the differential diagnosis of acute abdominal pain in children. We undertook a retrospective analysis of all the paediatric patients recorded as having had a laparoscopic Cholecystectomy performed at Prince Charles Hospital. 8 paediatric patients underwent Laparoscopic cholecystecomy between 2000 and 2008 consisting of $5 \mathrm{fe}-$ male patients and 3 male patients. The average age of the cohort was 14.1 years [12-16]. Premorbid obesity was a feature in 4 patients and all patients reported high fat diet. Abdominal UItrasound used to assess all 8 patients who presented with acute abdomen showed gallstones to be present in all. 7 patients underwent an elective procedure 3-6 months after the initial diagnosis was made and 1 patient had laparoscopic Cholecystectomy within 72 hours of initial presentation. 1 patient was found to have an inherited haematological disorder and 2 of the patients were sisters with a family history of gallstone disease. 2 patients presented with acute pancreatitis. Gallstone related cholecystitis is a rare occurrence amongst paediatric patients and is often overlooked as a differential diagnosis. We report 8 patients over an 8 year period. Conclusion: It is important that clinicians include cholecystitis and biliary colic in the differential diagnosis of patients presenting with acute abdomen in childhood not explained by other diagnoses. Laparoscopic Cholecystectomy is the treatment of choice and has minimal complications.
\end{abstract}

Keywords: Gallstones; Cholecystitis; Paediatrics; Laparoscopic Cholecystecomy

\section{INTRODUCTION}

Gallstones in children are rare but can become a potentially serious condition [1]. It does not always present itself in the classical clinical picture of adult gallstones and is not considered as a typical differential diagnosis of abdominal pain $[1,2]$. Right upper quadrant pain, nausea and vomiting may not always be present and initial diagnosis may be overlooked or delayed. We present our experience as a paediatric surgical team in managing Paediatric gallstones at a District General Hospital in South Wales. In order to assess how common paediatric gallstones are at a typical District general Hospital we re- viewed our patient records was carried at Prince Charles Hospital to identify any paediatric patient (aged 16 years or under) who had undergone a Cholecystectomy. Theatre reports and full case history notes were reviewed and data was extracted accordingly. Correspondence were also re- viewed to note the outcome of any subsequent follow up and to identify any other medical problems which may have arisen after the procedure or had an impact on the patient developing gallstones. All 8 patients underwent a laparoscopic Cholecystectomy performed by a single Consultant Paediatric Surgeon (Mr Asal Y Izzidien) at Prince Charles Hospital, Merthyr Tydfil.

\section{THE PATIENT COHORT}

All patients presented with abdominal pain but only 3 (37.5\%) patients localised this pain in the right upper quadrant. All patients eventually underwent Abdominal Ultrasound examination (USS) which showed the presence of gallstones. 3 patients underwent a Magnetic Retrograde Cholangio-Pancreotography and 1 patient underwent a HAIDA scan to assess gallbladder function. Every patient had a full history work up including a family history screen. Blood tests were assessed in each patient for the signs of haemolytic abnormalities. 2 patients $(25 \%)$ presented with pancreatitis and 1 patient had a known hereditary haemolytic condition (hereditary spherocytosis). This patient presented with jaundice and had an obstructive biochemical pattern.

Appendicitis was the initial working diagnosis in 3 of our patients and a Urinary tract Infection was suspected 
and treated empirically in another 2 patients.

Following diagnostic confirmation of gallstones, all 8 patients underwent laparoscopic Cholecystectomy. 7 patients underwent the procedure electively 4-6 months after the initial diagnosis was made and one patient had the procedure within 72 hours of initial presentation. When we reviewed the histopathology of our sample all 8 patients had evidence of acute cholecystitis changes in their gallbladder with oedematous, thick walled gallbladders. Stones were analysed and in 7 patients the stones were reported as being mixed cholesterol pigment stones. 1 patient had pigmented stones in keeping with their hereditary haemolytic condition.

Our only short term complication was one patient who developed an infection in his umbilicus portal site scar. This was treated with Augmentin 250/62 mg tds. He recovered and was discharged without any further problems. One patient developed keloid at one of her scar sites although no further action was taken and the patient was discharged.

\section{DISCUSSION}

Paediatric gallstones and pancreatitis are rare occurrence and in most cases, caused by trauma or chronic illness [1-3]. Gallstone related disease can often present in a non-specific manner and the classical signs are often absent. Murphy's sign is unreliable and children may find it difficult to describe the typical pain of biliary colic and cholecystitis. Clinicians often misdiagnose the condition as a Urinary tract infection and Appendicitis is also commonly documented in differential diagnoses $[1,2]$. Usually there is a chronic history of generalised abdominal pain of some months or more. In several cases, this had not been followed up to identify an underlying cause. Clinicians should therefore hold an index of suspicion for a diagnosis of gallstones in any paediatric presentation of abdominal pains, raised White cell count and fever [3].

Patients should undergo screening and evaluation for hereditary haemolytic conditions [1] as an increased breakdown of haemoglobin can produce large amounts of pigmented gallstones. Other underlying causes such as obesity, hypercholesterolemia, chronic liver disease and Cystic fibrosis may also be the primary cause of paediatric gallstones and should be excluded. There may be also a need to investigate for genetic predisposition as 2 of the cases were sisters and had strong family history of gall stones.

In the paediatric group non-invasive investigations should be the preferred choice if possible. Abdominal Ultrasound provides an effective method of identifying gallstones $[2,4]$. MRCP may be used to exclude stones in the common bile dict [5].

Weight loss is almost always advised in adult patients and the same rule appears to hold true for the paediatric population. Most of our patients were obese and being overweight is recognised as a risk factor for developing gallstones. Modern diets are often high in fat and with an obesity epidemic being predicted by some corners of medical society, the medical profession needs to be aware that gallstones are likely to become more common in children in years to come. Other factors associated with gallstones include use of the Oral contraceptive pill [4] and pregnancy at an earlier age may also contribute to a rise on paediatric gallstones in years to come.

\section{CONCLUSIONS}

There is increase in the incidence of gallstones in the paediatric age group possibly due to the worldwide epidemics of obesity but genetic predisposition may be a factor that needs more study to explore. Our study shows that the condition remains an uncommon presentation but should be considered in children presenting with abdominal symptoms, especially when other diagnoses have been excluded. Laparoscopic Cholecystectomy app- ears to be and appropriate and safe procedure to carry out on paediatric patients and has a low incidence of morbidity. It should therefore be considered as the procedure of choice in paediatric patients $[6,7]$ with gallstones. Due to early development of gall stones, hence the longer span of this pathology, paediatric patients may be more prone to developing complications including pancreatitis. It is paramount to prevent this condition as this carries a high risk of morbidity and mortality in the paediatric cohort $[5,8,9]$. We advocate carrying out surgery at the earliest opportunity. However, decisions should be taken on an individual basis if the clinical status of the patient dictates that more immediate surgery would be appropriate. At present our hospital has not performed Endoscopic procedures on paediatric patients and cases are typically referred to tertiary centres. However, if the incidence of paediatric gallstones continues to rise in line with childhood obesity, the likelihood of having to performing Endoscopic retrograde Cholangio Pancreatography (ERCP) will increase. This will in itself will have implications for service provision, patient safety and long term implications for patients.

\section{ACKNOWLEDGEMENTS}

D. S. carried out the relevant literature review and wrote the paper. $\mathrm{He}$ also analysed the patient data and reviewed the patient notes. N. N. and A. I. reviewed the paper and critically analysed the data. A. I. performed all the operative procedures

\section{REFERENCES}

[1] Sutton, R. and Cheslyn-curtis, S. (2001) Acute gallstone pancreatitis in childhood. Ann R Coll Surg Engl, 83, 406408. 
[2] Bruck, S.W., Sigmund, H.E., Rocchi, C. and Kim, P.C.W. The management of nonpigmented gallstones in children. Journ Paediatr Surg, 35, 729-732.

[3] Davenport, M. (2003) Laparoscopic surgery in children review. Ann R Coll Surg Engl, 85, 324-330.

[4] Haddock, G., Coupar, G., Youngson, G.G., MacKinlay, G.A. and Raine, P.A. (1994) Acute pancreatitis in children: A 15-year review. J Paediatr Surg, 29, 719-22

[5] Lerner, A., Branski, D. and Lebenthal, E. (1996) Pancreatic diseases in children. Paediatr Clin North Am, 43, 125 56.

[6] De Caluwe, D., Akl, U. and Corbally, M. Cholecystectomy verus cholecystolithotomy for cholelithiasis in childhood: Long-term outcome. Journ Paedr Surg., 36(10), 15181521.

[7] St Peter, S.D., Keckler, S.J., Nair, A., Andrews, W.S., Sharp, R.J. et al. (2008) Laparascopic cholecystectomy in the paediatric population. $J$ Laparoendosc Adv Tech A., 18(1), 127-30.

[8] Weizman, Z. and Durie, P.R. (1988) Acute pancreatitis in childhood. J Paediatr, 113, 24-9

[9] Lowe, M.E. and Greer, J.B. (2008) Pancreatitis in children and adolescents. Curr Gastroenterol Rep., 10(2), 128-35. 